\title{
ESPOROTRICOSE EM PACIENTE PORTADOR DE LÚPUS ERITEMATOSO SISTÊMICO
}

\section{SPOROTRICOSE IN PATIENTS WITH LUPUS ERYTHEMATOSO SISTEMICO}

\author{
Paula Felix Araujo ${ }^{1}$, Pillar Souza Viana Cancioº ${ }^{1}$ Luís Filipe Oliveira Silva', Leonel Raposo Motta ${ }^{1}$ \\ ${ }^{1}$ Acadêmico de Medicina da Faculdade de Medicina de Campos.
}

\section{RESUMO}

O presente caso clinico visa relatar paciente portadora de lúpus eritematoso sistêmico (LES), que adquiriu esporotricose na sua forma cutâneo linfática, e ela não pode ter a terapia preconizada para esses casos, pois ela é uma paciente com história de nefrite lúpica e o fármaco de primeira escolha é nefrotóxico, sendo assim foi utilizado a segunda opção de tratamento associada a antibióticos pois ela já havia adquirido infecção secundária, levando em conta o fato da paciente ser imunocomprometida, chegou-se a decisão de amputação da falange distal onde estava a ferida.

Palavras chave: Esporotricose; Lúpus eritematoso sistêmico; Paciente imunodeprimida

\begin{abstract}
The present clinical case aims to report a patient with systemic lupus erythematosus (SLE), who acquired sporotrichosis in its lymphatic cutaneous form, and she cannot have the therapy recommended for these cases, as she is a patient with a history of lupus nephritis and the drug first choice is nephrotoxic, so the second treatment option associated with antibiotics was used because she had already acquired secondary infection, taking into account the fact that the patient is immunocompromised, the decision was made to amputate the distal phalanx where the wound was. Key words: Sporotrichosis; Systemic lupus erythematosus; Immunodepressed patient
\end{abstract}


INTRODUÇÃO

O lúpus eritematoso sistêmico (LES) é uma doença inflamatória crônica multissistêmica, de característica autoimune e de etiologia desconhecida, relacionada a fatores genéticos, ambientais e hormonais e tende a ser mais comum e mais grave na raça negra e em asiáticos ${ }^{1}$. Pode ocorrer em pessoas de qualquer idade, raça e sexo, porém mulheres são mais acometidas. Ocorre predominantemente entre 20 e 45 anos e os sintomas podem surgir em diversos órgãos de forma lenta e progressiva ou mais rapidamente ${ }^{2}$. É uma doença pleomórfica com ampla variabilidade fenotípica de apresentação, gravidade e curso clínico e evolui habitualmente com períodos de atividade e de remissão. A maioria dos pacientes tem um curso relativamente benigno, porém a sobrevida global é menor quando comparada à da populaçãogeral ${ }^{3}$.

Os fatores genéticos, quando existem, promovem maior susceptibilidade a desenvolver a doença. O tipo de sintoma desenvolvido depende do tipo de autoanticorpo que o paciente possui. Por isso, cada pessoa com lúpus tende a se manifestar (sintomas) de maneiras específicas e muito pessoais, que variam em intensidade de acordo com a fases de atividade e remissão da doença ${ }^{2}$.

O LES é uma doença potencialmente grave. Sendo uma vasculite de médios e de pequenos vasos, todos os órgãos estão sujeitos a danos. No entanto, com advento do arsenal terapêutico e de diagnóstico precoce, as crises agudas da doença podem ser melhor controladas; a mortalidade por causa da vasculite diminuiu, e a doença assumiu um caráter mais crônico' ${ }^{1}$.

O tratamento da pessoa com LES depende do tipo de manifestação apresentada e deve, portanto, ser individualizado ${ }^{2}$. As principais causas de morte de um paciente lúpico são: infecção, atividade da doença, doença cardiovascular, lesão renal e câncer ${ }^{3}$.

Pacientes com LES parecem possuir um aumento no risco para infecções. Entre os defeitos do sistema imune, incluem-se a redução de linfócitos T CD4+, a deficiência de componentes do sistema complemento, a neutropenia e a linfopenia. As infecções são uma das principais causas de morbimortalidade nessa doença, sejam elas precoces ou tardias, e todos os esforços devem ser dirigidos para sua prevenção e tratamento'.

O risco de infecções no LES é maior pelo uso de imunossupressores e particularmente pelos glicocorticoides, que são regularmente utilizados para tratamento das complicações sistêmicas da doença.9 Essa última medicação ainda não possui a especificidade desejada, que seria a de atuar somente sobre células autorreativas e disfuncionais, e acaba por minar as defesas normais do organismo, favorecendo a agressão por microrganismos ${ }^{1}$.

Já a esporotricose é uma micose subcutânea causada pelo fungo Sporothrix schenckii que afeta principalmente homens e animais. É a mais frequente das micoses subcutâneas. Os fungos do complexo Sporothrix sp vivem no solo e se multiplicam em matéria orgânica em decomposição ${ }^{4}$. O fungo é amplamente disperso na natureza, justificando assim a sua distribuição geográfica cosmopolita da doença. Este fungo tem sido descrito em todos os países do mundo como um organismo comumente encontrado em vegetais esolo 5 .

A doença é considerada endêmica na América Latina. É apontada como a micose sucbutânea mais comum na América do Sul. No que tange ao Brasil, apresenta-se de forma endêmica na região Sudeste do país, nos últimos anos, a esporotricose no estado do Rio de Janeiro (RJ) tem sido relacionada com a doença em gatos. Milhares de casos humanos e felinos foram diagnosticados no RJ nas duas últimas décadas. É caracterizada por lesões nodulares que podem supurar ou ulcerar. Tem evolução subaguda ou crônica na maior parte dos casos ${ }^{4}$.

A esporotricose pode acometer o ser humano de ambos os sexos, sem predileção por faixa etária ou raça, independe de fatores individuais predisponentes. 0 modo de transmissão clássico ocorre pela inoculação traumática do fungo na pele, sendo, por vezes, classificada como doença de cunho ocupacional para floristas, agricultores, jardineiros, caçadores, veterinários, tendo estes maiores riscos à infecção ${ }^{4}$. Entre os anos de 1998 a 2001 foram descritos 178 casos da doença, no Rio do Janeiro, sendo considerada a epidemia mais relevante por transmissão zoonótica no mundo. Já entre os anos de 2002 a 2004, uma casuística ainda maior, com 572 casos da doença, sendo a transmissão zoonótica por gatos domésticos retificadora da epidemia iniciada em $1998^{5}$.

A apresentação clínica da doença é muito variada, observa-se desde nódulos isolados a lesões múltiplas disseminadas. A forma clínica 
apresentada, após a contaminação, depende de diversos fatores, como a carga do inóculo, a profundidade da inoculação traumática, a tolerância térmica da cepa e a resposta imunológica do hospedeiro. As lesões costumam ser restritas a pele, tecido celular subcutâneo e vasos linfáticos adjacentes. Em raras ocasiões, podem disseminarse para outros órgãos, ou ainda ser primariamente sistêmica ${ }^{4}$.

O diagnóstico da esporotricose é baseado na história clínica e no isolamento do fungo, através de cultura. Existem diversas modalidades de tratamento para esporotricose, desde o calor local ao uso da solução saturada de iodeto de potássio e de antifúngicos como Itraconazol, com taxas de sucesso terapêutico variáveis na literatura médica. Embora não seja doença de notificação compulsória, estudos recentes e dados de institutos de pesquisa mostram que, nos últimos anos, tem havido aumento no número de casos de esporotricose entre animais domésticos em zonas urbanas, como no Rio de Janeiro ${ }^{4}$.

\section{OBJETIVO}

Relatar um caso de Esporotricose na forma cutâneo-linfática em uma paciente imunodeprimida, no município de Campos dos Goytacazes, estado do Rio de Janeiro. Este relato visa mostrar que neste caso, em especial, a paciente não teve a terapia preconizada que seria a Polimixina B, pois ela é uma paciente com história de nefrite lúpica e este fármaco é nefrotóxico.

\section{DESCRIÇÃO}

Paciente sexo feminino, 33 anos, solteira, branca, natural de Campos dos Goytacazes (RJ), portadora de Lúpus Eritematoso Sistêmico em acompanhamento ambulatorial de rotina na reumatologia no Hospital Escola Álvaro Alvim, apresentando diminuta lesão nodular ulcerada em 2 o quirodáctilo esquerdo, iniciado assim empiricamente antibiótico e antifúngico, o exame físico apresentava-se sem alterações nesta consulta. Cerca de um mês neste esquema, paciente com evolução do quadro, e com surgimento de cordão endurecido em direção aos linfonodos da região, linfonodomegalias em MSE, acompanhada de intensas dores e progressão da lesão. Com isso, a paciente foi admitida na enfermaria de Clínica Médica do HEAA. Baseada na anamnese e história positiva de exposição anterior a felino com infecção pelo fungo Sporothrix spp, foi feito diagnóstico clínico da forma cutâneo-linfática da esporotricose. A paciente apresenta comprometimento renal secundário ao lúpus, nefrite lúpica, história de TVP em MIE, atualmente em uso regular de micofenolato de mofetil $06 \mathrm{cp} /$ dia, prednisona $20 \mathrm{mg} /$ dia e hidroxicloroquina $400 \mathrm{mg} / \mathrm{dia}$, foi optado por manter o esquema inicial, devido ao acometimento renal da paciente, a equipe seguiu por uma conduta mais conservadora, descartando a polimixina B por sua nefrotoxicidade, mas seria o preconizado para o caso, devido a imunossupressão da paciente, porém foi feita otimização da dose do antifúngico, intraconazol $200 \mathrm{mg} /$ dia por 10 dias. Após segundo dia de internação hospitalar paciente realizou procedimento de desbridamento da lesão, onde foi colhido material para realização de cultura, e o swab de secreção acusava crescimento de Pseudomonas sp sensível a tazocin, ou seja, lesão secundaria se iniciou, com isso a equipe de cirurgia e clínica médica determinou que era aconselhável a amputação da falange distal de 2 o quirodáctilo esquerdo, no sexto dia de internação hospitalar. Após o final do esquema terapêutico proposto e cirurgia paciente evoluiu bem, tendo alta hospitalar, e continuará acompanhamento habitual na reumatologia.

\section{CONCLUSÃO}

Diante do exposto, fica evidente que o diagnóstico da Esporotricose pode vir associado a outras doenças autoimunes, como o Lúpus Eritematoso Sistêmico. Além disso, deve ser observado o cuidado em relação à terapia medicamentosa, uma vez que a nefrite lúpica restringe algumas opções terapêuticas. Portanto, é imprescindível uma análise criteriosa de determinados casos para evitar intervenções que resultem em sequelas prejudiciais.

\section{REFERÊNCIAS}

Skare Thelma Larocca, Dagostini Jéssica Scherer, Zanardi Patrícia Imai, Nisihara Renato Mitsunori. Infecções e lúpus eritematoso sistêmico. Einstein (São Paulo) [Internet]. 2016 Mar [citado em 201928 de junho]; 14 (1): 47-51. Disponível em: http://www.scielo.br/scielo.php?script=sci_arttext\&pid=S1679-45082016000100047\&lng=en. 
SBR - Sociedade Brasileira de Reumatologia. Lúpus Eritematoso Sistêmico (LES). Cartilha da SB. 2011

Klumb, E.M.; Silva, C.A.; Lanna, C.C.; SATO, I.; Borba, E.; Brenol, J. C.; Albuquerque, E. M.; Monticielo, O. A.; Costallat, L. T.; Latorre, L. C.; Sauma, M. F.; Bonfá, E. S.; Ribeiro, F. M. Consensus of the Brazilian Society of Rheumatology for the diagnosis, management and treatment of lupus nephritis. Revista Brasileira de Reumatologia (English Edition), Volume 55, Issue 1, 2015, pp. 1-21

Secretaria de Estado de Saúde do Rio de Janeiro. Boletim epidemiológico esporotricose 001/2018. Vigilância e cenário epidemiológico: esporotricose no estado do rj. Período de 2015 a 2018. Rio de Janeiro, RJ. Maio de 2018. MUNIZ A.S.; PASSOS J. P. Esporotricose humana: conhecendo e cuidando em enfermagem. Artigo de Revisão. Rev. enferm. UERJ, Rio de Janeiro, 2009 abr/jun; 17(2):268-72. 